\title{
Maus-tratos infantis no currículo dos cursos de Odontologia do Rio de Janeiro
}

\author{
Alice Pinheiro Costa*; Rachel Lima Ribeiro Tinoco** \\ * Graduada em Odontologia, Universidade Salgado de Oliveira \\ ** Mestre em Odontologia Legal, Professora de Odontologia \\ Legal e Deontologia, Universidade Salgado de Oliveira
}

Recebido em 09/01/2019. Aprovado em 26/05/2019.

\begin{abstract}
RESUMO
Os maus-tratos infantis são vistos como um dos maiores problemas de saúde pública de acordo com a Organização Mundial da Saúde e, no âmbito odontológico, o cirurgião-dentista pode influenciar diretamente no diagnóstico e denúncia desses casos, desde que possua a percepção e as informações necessárias. O presente estudo teve por objetivo avaliar se o currículo dos cursos de Odontologia do Rio de Janeiro fornece conhecimento e preparo suficiente para os acadêmicos acerca dos maus-tratos infantis. Para tanto, foi realizada uma pesquisa de campo com coleta de dados por meio da aplicação de um questionário semiestruturado em oito cursos de Odontologia do Rio de Janeiro abrangendo 200 estudantes do último ano da graduação. Destes, 53 (26,5\%) eram de instituições públicas e 147 $(73,5 \%)$ de instituições particulares. Dentre os participantes, $74,5 \%$ disseram que a temática foi abordada no curso, principalmente nas disciplinas de Odontologia Legal e Odontopediatria. Todos afirmaram a importância do tema na graduação e apenas $29(14,5 \%)$ não acreditam ser de responsabilidade do cirurgião-dentista diagnosticar esses casos. Quanto ao conhecimento do Estatuto da Criança e do Adolescente, 129 (64,5\%) estudantes afirmaram conhecer, entretanto 107 (53,5\%) desconhecem as implicações legais para os cirurgiões-dentistas. Hematomas, retração da criança e medo foram os sinais e sintomas mais citados pelos estudantes. Quanto à conduta, a maioria afirmou que comunicaria ao Conselho Tutelar. Conclui-se que os acadêmicos de Odontologia da amostra possuem um conhecimento regular sobre o tema maus-tratos infantis.
\end{abstract}

Descritores: Violência. Criança. Educação em Odontologia. Odontologia Legal. Estudantes de Odontologia.

\section{INTRODUÇÃO}

Os maus-tratos infantis são vistos como um grave problema de saúde pública, de acordo com a
Organização Mundial da Saúde (OMS)1. Essa violência é de natureza complexa, por atingir a saúde e a qualidade de vida desses indivíduos, 
afetando muitas vezes seus direitos fundamentais, como acesso à educação, à saúde e a cuidados para seu desenvolvimento ${ }^{2}$. Quatro tipos de maus-tratos são usualmente conhecidos: abuso físico, abuso sexual, abuso psicológico e negligência ${ }^{3}$.

O Código Penal Brasileiro ${ }^{4}$, no artigo 136, conceitua o crime de maus-tratos como "Expor a perigo a vida ou a saúde de pessoa sob sua autoridade, guarda ou vigilância, para fim de educação, ensino, tratamento ou custódia, quer privando-a de alimentação ou cuidados indispensáveis, quer sujeitando-a a trabalho excessivo ou inadequado, quer abusando de meios de correção ou disciplina".

O Estatuto da Criança e do Adolescente (ECA) descreve maus-tratos em seu artigo 232, como "submeter criança ou adolescente sob sua autoridade, guarda ou vigilância a vexame ou constrangimento" . Conforme estabelece a mesma lei, em seu artigo 245, o responsável por estabelecimento de atenção à saúde tem obrigação de comunicar à autoridade competente casos suspeitos ou confirmados. O Ministério da Saúde, por meio da Portaria 1.968 , de 25 de outubro de 2001, instituiu formulário próprio para notificação destes casos atendidos nos serviços do Sistema Único de Saúde, e os incluiu na lista de notificação compulsória na Portaria 104, de 25 de janeiro de 2011.

No Brasil as primeiras denúncias sobre os maus-tratos infantis se deram na década de $1970^{6}$, entretanto a atenção para esse problema social ocorreu somente nos anos 1980, com a criação de associações e centros de proteção à criança. $\mathrm{Na}$ década seguinte o ECA foi promulgado, sendo considerado um avanço na defesa e proteção de crianças e adolescentes, prevendo inclusive a obrigatoriedade da denúncia por parte de profissionais da área da saúde ao Conselho Tutelar da respectiva localidade, em caso de suspeita ou confirmação de maus-tratos ${ }^{7}$.

Nesse sentido, o cirurgião-dentista se encontra em posição favorável para diagnosticar e denunciar os possíveis casos de maus-tratos, já que $50 \%$ das lesões ocorrem na região de cabeça, pescoço, face e boca ${ }^{8}$. Mesmo assim, alguns fatores levam os profissionais de saúde a se omitirem nesses casos, como o medo de perder pacientes, a incerteza do diagnóstico, do mecanismo de denúncia e o desconhecimento da verdadeira responsabilidade em denunciar, assim como o medo de se envolver legalmente ${ }^{9}$.

Além disso, pesquisas demonstram que a grande maioria dos cirurgiões-dentistas revela falta de conhecimento e de conduta padrão diante de casos suspeitos de maus-tratos infantis, e isso está normalmente associado à ausência ou insuficiência de informação obtida no curso de graduação ${ }^{6}$. Esse quadro indica a necessidade da capacitação do profissional, que pode ser obtida pela inclusão do assunto no currículo dos cursos de Odontologia.

Diante do exposto, considerando que os casos de maus-tratos infantis são crescentes, e que no Brasil, a cada ano, $12 \%$ das crianças com menos de 14 anos são vítimas de alguma forma de violência doméstica ${ }^{3}$, o cirurgião-dentista, como profissional presente na rotina de crianças e adolescentes, tem papel direto sobre o diagnóstico e denúncia desses casos. Assim, o presente estudo tem por objetivo analisar o conhecimento dos estudantes do último ano de graduação de cursos de Odontologia do Rio de Janeiro acerca do tema.

\section{MATERIAIS E METÓDOS}

O estudo apresentado refere-se a uma pesquisa de campo, observacional e seccional, em que o instrumento de coleta de dados foi um questionário (figura 1) semiestruturado com dez perguntas, sendo duas delas subjetivas e oito objetivas, relacionadas aos maus-tratos infantis e à abordagem do tema durante o curso de graduação.

A coleta de dados foi realizada em oito cursos de Odontologia do Estado do Rio de Janeiro, tendo como amostra 200 estudantes do último ano 
de formação. O questionário não contém campo para identificação do participante e foi separado do Termo de Consentimento Livre e Esclarecido (TCLE) assim que respondido. Uma versão eletrônica do referido questionário foi criada no Google Forms ${ }^{\circledR}$ (Google, San Francisco, EUA), com link para o TCLE em anexo para acesso dos participantes. O convite à pesquisa foi distribuído entre os estudantes de Odontologia, não só por abordagem direta, mas também nas redes sociais, razão pela qual não é possível precisar o número de pessoas que não aceitaram participar.

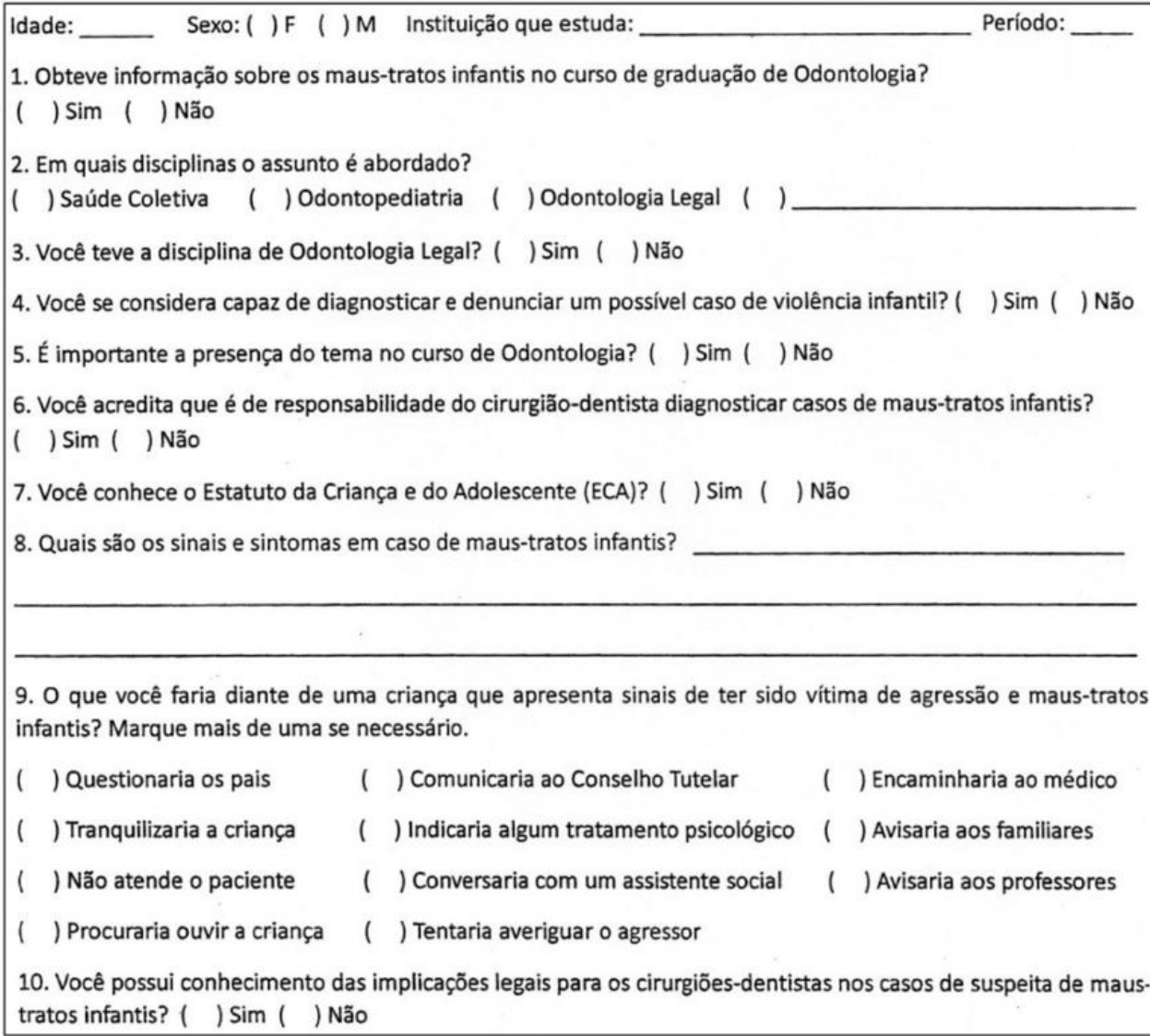

Figura 1. Questionário utilizado para coleta de dados

Os dados foram tabulados em planilha do software Excel® (Microsoft, Seattle, EUA) e avaliados por meio de estatísticas descritivas. O projeto foi submetido ao Comitê de Ética em Pesquisa (CEP) da Universidade Salgado de Oliveira e obteve aprovação, registrada no parecer
2.720.414 (CAAE: 88733318.7.0000.5289).

\section{RESULTADOS}

Participaram da pesquisa um total de 200 estudantes, com idade média de 24,18 $( \pm 4,26)$ anos, sendo 158 acadêmicos do gênero feminino 
(idade média $24,1 \pm 3,88$ ) e 42 do gênero masculino (idade média de 24,18 $\pm 5,21$ ). Dos participantes, $55(27,5 \%)$ estavam no $7^{\circ}$ período, $101(50,5 \%)$ no $8^{\circ}$ período e $44(22,0 \%)$ no $9^{\circ}$ período. Cabe esclarecer que os cursos têm duração entre 4 e 5 anos. Quanto à instituição de ensino, $53(26,5 \%)$ dos participantes pertenciam a instituições públicas e $147 \quad(73,5 \%)$ a instituições particulares (tabela 1).

Quando questionados se obtiveram informações sobre os maus-tratos infantis no curso de graduação em Odontologia, a maioria $(74,5 \%)$ dos acadêmicos respondeu positivamente, em diferentes disciplinas, relacionadas na figura 2.

Tabela 1. Distribuição dos participantes de acordo com gênero, idade (média \pm desvio padrão) e instituição

\begin{tabular}{|c|c|c|c|}
\hline Sexo & Masculino & Feminino & Total \\
\hline $\mathrm{n}(\%)$ & $42(21 \%)$ & $158(79 \%)$ & $200(100 \%)$ \\
\hline Idade (média \pm desvio padrão) & $24,1( \pm 5,21)$ & $24,18( \pm 3,88)$ & $24,18( \pm 4,26)$ \\
\hline \multicolumn{4}{|l|}{ Instituição de Ensino } \\
\hline Universidade Salgado de Oliveira** & 20 & 75 & $95(47,5 \%)$ \\
\hline Universidade Federal Fluminense* & 6 & 37 & $43(21,5 \%)$ \\
\hline Universidade do Grande Rio** & 8 & 20 & $28(14,0 \%)$ \\
\hline Universidade Veiga de Almeida** & 3 & 15 & $18(9,0 \%)$ \\
\hline Universidade Federal do Rio de Janeiro* & 2 & 7 & $9(4,5 \%)$ \\
\hline Faculdade São José*** & 2 & 3 & $5(2,5 \%)$ \\
\hline Universidade Estácio de Sá** & 1 & 0 & $1(0,5 \%)$ \\
\hline Universidade Estadual do Rio de Janeiro* & - & 1 & $1(0,5 \%)$ \\
\hline Total & 42 & 158 & $200(100 \%)$ \\
\hline
\end{tabular}



Figura 2. Disciplina em que o tema é abordado no curso de Odontologia 
Dos 200 estudantes, 197 (98,5\%) afirmaram ter tido a disciplina de Odontologia Legal durante a graduação. Quando foram questionados se foram capacitados para diagnosticar e denunciar um possível caso de violência infantil 161 (80,5\%) estudantes responderam positivamente. Todos concordaram com a importância do tema no curso de Odontologia, e a maioria $(85,5 \%)$ afirmou que é responsabilidade do cirurgião-dentista fazer $\mathrm{o}$ diagnóstico dos casos de maus-tratos infantis. Quando perguntados sobre o conhecimento das implicações legais para os cirurgiões-dentistas nos casos de suspeita de maus-tratos infantis, 107 $(53,5 \%)$ afirmaram desconhecê-las, mesmo com $129(64,5 \%)$ estudantes relatando o conhecimento do ECA (tabela 2).

Tabela 2. Respostas às perguntas fechadas do questionário aplicado

\begin{tabular}{lcccc}
\multicolumn{1}{c}{ Pergunta } & \multicolumn{2}{c}{ Sim } & \multicolumn{2}{c}{ Não } \\
& $\mathrm{n}$ & $\%$ & $\mathrm{n}$ & $\%$ \\
\hline $\begin{array}{l}\text { Obteve informação sobre os maus-tratos infantis no curso de } \\
\text { graduação em Odontologia? }\end{array}$ & 149 & $74,5 \%$ & 51 & $25,5 \%$ \\
Você teve a disciplina de Odontologia Legal? & 197 & $98,5 \%$ & 3 & $1,5 \%$ \\
Você se considera capaz de diagnosticar e denunciar um possível & 161 & $80,5 \%$ & 39 & $19,5 \%$ \\
caso de violência infantil? & & & & - \\
É importante a presença do tema no curso de Odontologia? & 200 & $100 \%$ & - & - \\
Você acredita que é de responsabilidade do dentista diagnosticar & 171 & $85,5 \%$ & 29 & $14,5 \%$ \\
casos de maus-tratos infantis? & & & & \\
Você conhece o estatuto da criança e do adolescente (ECA)? & 129 & $64,5 \%$ & 71 & $35,5 \%$ \\
Você possui conhecimento das implicações legais para os & 93 & $46,5 \%$ & 107 & $53,5 \%$ \\
cirurgiões-dentistas nos casos de suspeita de maus-tratos infantis? & & & & \\
\hline
\end{tabular}

Dentre as respostas às perguntas abertas sobre os sinais e sintomas de maus-tratos infantis, a presença de hematomas, a retração da criança e o medo foram os mais citados. Os sinais e sintomas foram contabilizados separadamente, e esta foi uma pergunta de resposta livre, sem restrição de número, ou indução com opções. Por este motivo, os valores percentuais não totalizam $100 \%$ (tabela 3).

Em relação ao que fariam diante de uma criança com sinais de agressão e maus-tratos, 161 $(80,5 \%)$ dos estudantes afirmaram que comunicariam ao Conselho Tutelar, 149 (74,5\%) procurariam ouvir a criança e 97 (48,5\%) conversariam com um assistente social. As respostas dos acadêmicos podem ser observadas na tabela 4, que não apresenta total de $100 \%$, uma vez que, nesta pergunta, os participantes puderam marcar mais de uma opção.

\section{DISCUSSÃO}

Dados revelam que anualmente no Brasil aproximadamente 10 milhões de crianças e adolescentes sofrem maus-tratos ${ }^{11}$, que ocorrem por uma combinação de fatores individuais, familiares e sociais ${ }^{12}$. A gravidade desse problema revela a necessidade do cirurgião-dentista em estar apto para identificar casos de violência infantil.

Os maus-tratos infantis são classificados como abuso físico, abuso psicológico, abuso sexual e negligência. $\mathrm{O}$ abuso físico se apresenta deixando marcas evidentes ou não no corpo. $\mathrm{O}$ abuso psicológico é o mais comum e mais difícil de ser identificado, prejudicando o crescimento e desenvolvimento do indivíduo maltratado. 
Tabela 3. Sinais e sintomas de maus-tratos infantis citados pelos participantes, listados por ordem de frequência de citação em pergunta aberta

\begin{tabular}{lcc}
\hline Sinais e sintomas & $\mathbf{n}^{*}$ & \%* \\
\hline Hematomas & 80 & 40,0 \\
Retração & 63 & 31,5 \\
Medo & 63 & 31,5 \\
Marcas corporais & 27 & 13,5 \\
Lesões físicas & 27 & 13,5 \\
Manchas no corpo & 18 & 9,0 \\
Timidez & 17 & 8,5 \\
Queimaduras & 14 & 7,0 \\
Comportamento & 11 & 5,5 \\
Mudança de comportamento & 14 & 7,0 \\
Machucados & 8 & 4,0 \\
Equimoses & 8 & 4,0 \\
Escoriações & 7 & 3,5 \\
Contradição na história contada & 6 & 3,0 \\
Agressividade & 6 & 3,0 \\
Ansiedade & 6 & 3,0 \\
Depressão & 5 & 2,5 \\
Laceração em lábio & 5 & 2,5 \\
Dificuldade de comunicação & 4 & 2,0 \\
Tristeza & 4 & 2,0 \\
Outros & 121 & 60,5 \\
\hline
\end{tabular}

Tabela 4. Conduta diante de uma criança que apresenta sinais de ter sido vítima de agressão e maustratos

\begin{tabular}{lcc}
\hline \multicolumn{1}{c}{ Conduta } & $\mathbf{n}^{*}$ & \%* $^{*}$ \\
\hline Comunicaria ao conselho tutelar & 161 & 80,5 \\
Procuraria ouvir a criança & 149 & 74,5 \\
Conversaria com um assistente social & 97 & 48,5 \\
Avisaria aos professores & 78 & 39,0 \\
Tranquilizaria a criança & 72 & 36,0 \\
Questionaria aos pais & 56 & 28,0 \\
Indicaria algum tratamento psicológico & 52 & 26,0 \\
Tentaria averiguar o agressor & 39 & 19,5 \\
Avisaria aos familiares & 28 & 14,0 \\
Não atende o paciente & 1 & 0,5 \\
\hline *Os valores percentuais desta tabela não totalizam 100\%, por se tratar de pergunta de múltipla \\
escolha, sem restrição de número de respostas
\end{tabular}

A violência sexual se dá por meio da indução violência física ou ameaça. Já a negligência é a de práticas eróticas e sexuais forçadas pela omissão de cuidados básicos à criança e ao 
adolescente por parte do responsável ${ }^{7}$. Todas essas formas de abuso podem ser reconhecidas por cirurgiões-dentistas desde que possuam a capacitação correta, o que motivou o presente estudo, cujos resultados obtidos podem ser de grande valia para elaboração de novas propostas, e mapeamento de possíveis lacunas na formação do profissional da Odontologia.

A partir da coleta de dados e resultados desse estudo pode-se verificar que a maioria dos participantes era do sexo feminino e com idade média de 24,1. O gênero feminino também foi predominante em outros estudos como nos de Sousa et al. $(2012)^{8}$ e Silva et al. $(2015)^{13}$.

A maioria dos acadêmicos afirmaram ter recebido informações sobre os maus-tratos infantis durante a graduação. Resultados semelhantes foram encontrados na pesquisa de Sousa et al. $(2012)^{8}$ em que $89,8 \%$ dos 59 participantes, do Centro Universitário de João Pessoa (PB) afirmaram haver recebido informações durante a graduação sobre o tema. Entretanto no estudo de Silva et al. $(2015)^{13}$ com amostra de estudantes da Universidade Federal do Espírito Santo, apenas $26 \%$ dos 100 entrevistados - acadêmicos do $5^{\circ}$ ao $9^{\circ}$ período - receberam informações sobre o assunto, tendo os autores concluído haver deficiência no conteúdo tratado durante o curso. Em relação à disciplina em que o tema foi abordado, assim como no estudo de Matos et al. $(2013)^{9}$, a mais citada foi a Odontologia Legal, por $49(24,5 \%)$ estudantes.

A maioria $(80,5 \%)$ dos pesquisados informou ser capaz de diagnosticar e denunciar um possível caso de violência infantil. Dentre estes, 161 acadêmicos informaram que comunicariam ao Conselho Tutelar. Resultados semelhantes foram encontrados no trabalho de Serpa e Ramos (2011) ${ }^{6}$, com 75,8\% mencionando a necessidade de denunciar ao Conselho Tutelar. Cabe ressaltar, entretanto, que esta informação foi coletada por meio de pergunta objetiva, com opções a serem marcadas, o que não necessariamente se repetiria em perguntas abertas.

No presente estudo todos os participantes responderam positivamente quando questionados sobre a importância do tema no curso de Odontologia. A violência infantil não é somente um problema social. Por sua amplitude é vista também como um problema de saúde pública, se inserindo nos serviços de saúde dos quais o cirurgião-dentista faz parte $^{14}$, podendo assim ter influir na redução desses acontecimentos.

Quando questionados se é responsabilidade do cirurgião-dentista diagnosticar casos de maustratos infantis, verificou-se que $85,5 \%$ dos acadêmicos responderam positivamente, dado bem semelhante ao encontrado por Serpa e Ramos $(2011)^{6}$. O cirurgião-dentista, principalmente o Odontopediatra, atendendo de maneira cuidadosa e atenta, pode identificar características físicas e emocionais do abuso infantil ${ }^{15}$.

O ECA contribui de maneira significante com informações sobre o tema e prevê, em seu Artigo 13, a obrigatoriedade de comunicar ao Conselho Tutelar da respectiva localidade os casos de suspeita ou confirmação de maus-tratos a crianças e adolescentes ${ }^{5}$. Porém, 35,5\% dos pesquisados informaram não ter conhecimento acerca do mesmo.

Em relação aos sinais e sintomas, nesse estudo a maioria dos participantes citou lesão física (hematomas) e alterações de comportamento, como a retração da criança e o medo, convergindo com o estudo de Silva et al. $(2015)^{13}$ em que esses resultados foram bem semelhantes. Estudos evidenciam os hematomas em diversos estágios de cura como ferimentos comumente relacionados ao abuso físico ${ }^{16}$. Já a retração da criança e o medo podem ser decorrentes de diversas formas de violência infantil ${ }^{17}$.

É válido destacar que embora os pesquisados tenham citado de forma correta os sinais e sintomas dos maus-tratos, a negligência que é a forma mais 
comum de maus-tratos contra crianças e adolescentes $^{18}$ não foi abordada pelos estudantes. Assim, torna-se evidente a necessidade de maior atenção para esse assunto nos cursos de Odontologia.

Dentre os participantes, $53,5 \%$ relataram desconhecer as implicações legais para os cirurgiões-dentistas que não notifiquem casos suspeitos de maus-tratos infantis. Resultado semelhante foi encontrado por Matos et al. (2013) ${ }^{9}$, estudo no qual $49 \%$ dos graduandos também afirmaram desconhecer as implicações legais. $\mathrm{O}$ Artigo 245 do ECA estabelece pena de multa de três a vinte salários de referência, aplicando-se o dobro em caso de reincidência, para o profissional que deixar de comunicar à autoridade os casos de que tenha conhecimento ${ }^{5}$.

A violência infantil é de extrema complexidade e diante dos dados obtidos neste estudo fica clara a necessidade de sua abordagem nos cursos de graduação, cumprindo lamentar a ausência do assunto no currículo de $25,5 \%$ dos pesquisados. Assim, o estudo contribuiu para fornecer um quadro do futuro cirurgião-dentista fluminense quanto à capacitação no tema e de sua importância. Entretanto, mais pesquisas na área precisam ser realizadas com o intuito de falar cada vez mais sobre o assunto, divulgando os resultados e buscando a diminuição desses casos. Além disso, é fundamental que as associações de professores especializados em Odontologia Legal e em Odontopediatria ofereçam capacitação no conteúdo.

\section{CONCLUSÃO}

Conclui-se que, de maneira geral, os estudantes do último ano de Odontologia entrevistados apresentam conhecimento parcial do tema. A maioria afirmou ter tido informações durante a graduação, ser capaz de diagnosticar e denunciar casos de violência infantil e ainda citar corretamente sinais e sintomas de violência infantil. Porém, muitos destes ainda desconhecem o Estatuto da Criança e do Adolescente e os aspectos legais referentes aos cirurgiões-dentistas. Sugere-se que o tema seja abordado de maneira interdisciplinar nos cursos de Odontologia para que se formem dentistas capazes de contribuir efetivamente no combate à violência infantil.

\section{ABSTRACT \\ Child maltreatment in the curriculum framework of the Dentistry courses in Rio de Janeiro}

Child maltreatment is seen as one of the major public health problems according to the World Health Organization and the dentist can directly influence the diagnosis and make a denunciation of these cases, if having the knowledge and training needed. The present study aimed to assess whether the curriculum framework of the Rio de Janeiro's Dentistry courses provides enough knowledge and preparation to the academics about child maltreatment. For that, a field survey was conducted with data collection through the application of a semi-structured questionnaire in eight dental courses in Rio de Janeiro, totalizing a sample of 200 students in the last year of graduation. Of these, $53(26.5 \%)$ belong to public institutions and $147(73.5 \%)$ to private institutions. Among the participants, $74.5 \%$ said that the theme was addressed in the course, mainly in the disciplines of Legal Dentistry and Pediatric Dentistry. All of them affirmed the importance of the topic at graduation and only 29 (14.5\%) did not believe it was among the dentist responsibility to diagnose these cases. Regarding the knowledge of the Child and Adolescent Statute (CAS), 129 $(64.5 \%)$ students stated to know, while 107 $(53.5 \%)$ ignore the legal implications for dentists. Bruises, fear and retraction of the child were the most cited signs and symptoms. As for the conduct, the majority said they would report to the Guardianship Council. It was concluded that the dental students in the sample have a regular knowledge about this subject.

Descriptors: Violence. Children. Dental Education. Forensic Dentistry. Dentistry Students. 


\section{REFERÊNCIAS}

1. Brasil. Ministério da Saúde. Linha de cuidado para a atenção integral à saúde de crianças, adolescentes e suas famílias em situação de violências: Orientação para gestores e profissionais de saúde. Brasília: Ministério da Saúde, 2010.

2. Associação Brasileira Multiprofissional de Proteção à Infância e à Adolescência. Maus tratos contra crianças e adolescentes: proteção e prevenção. Guia de orientação para profissionais de saúde. 2nd ed. Rio de Janeiro: Petrópolis; 1997.

3. Gomes LS, Pinto TCA, Costa EMMB, Ferreira JMS, Cavalcanti SDLB, GranvilleGarcia AF. Percepção de acadêmicos de odontologia sobre maus-tratos na infância. Odontol Clín-Cient. 2011;10(1):73-8.

4. Brasil. Código Penal Brasileiro. Decreto-Lei $\mathrm{n}^{\circ} 2.848$, de 7 de dezembro de 1940.

5. Brasil. Estatuto da Criança e do Adolescente. Lei no 8.069, de 13 de julho de 1990.

6. Serpa EM, Ramos AAS. Percepção dos maus tratos infantis pelos estudantes de odontologia da UFPB. Int J Dent. 2011;10(4):234-41.

7. Brasil. Ministério da Saúde. Notificação de maus-tratos contra crianças e adolescentes pelos profissionais de saúde. Um passo a mais na cidadania em saúde. Série A. N. 167, Brasília: Ministério da Saúde; 2002.

8. Sousa GFP, Carvalho MMP, Garcia AFC, Gomes MNC, Ferreira JMS. Conhecimento de acadêmicos em odontologia sobre maus-tratos infantis. Odonto. 2012;20(40):109-17.

9. Matos FZ, Borges ÁH, Mamede Neto I, Rezende CD, Silva KL, Pedro FLM, et al. Avaliação do conhecimento dos alunos de graduação em Odontologia $\mathrm{x}$ cirurgião dentista no diagnóstico de maus-tratos a crianças. Rev Odontol Bras Central. 2013;22(63):153-7.
10. Biss SP, Duda JG, Tomazinho PH, Pizzatto E, Losso EM. Maus tratos infantis: avaliação do currículo dos cursos em odontologia. Rev ABENO. 2015;15(1): 55-62.

11. Universidade Positivo. Maus-tratos infantis. $\mathrm{O}$ papel dos Cirurgiões-Dentistas na proteção das crianças e adolescentes. Proteção e prevenção de maus-tratos infantis. Curtitiba: Universidade Positivo; 2015.

12. Cavalcanti AL. Abuso infantil: protocolo de atendimento odontológico. Rev Bras Odont. 2001;58(6):378-80.

13. Silva Júnior MF, Pagel MD, Campos DMKS, Miotto MHMB. Conhecimento dos acadêmicos de Odontologia sobre maus-tratos infantis. Arq Odontol. 2015;51(3):138-44.

14. Tornavoi DC, Galo R, Silva RH. Conhecimento dos profissionais de Odontologia sobre violência doméstica. RSBO. 2011;8(1):54-9.

15. Alves PM, Cavalcanti AL. Diagnóstico do abuso infantil no ambiente odontológico. Uma revisão de literatura. Publ UEPG Ci Biol Saúde. 2003;9(3/4):29-35.

16. Massoni AC LT, Ferreira ÂMB, Aragão AKR, Menezes VA, Colares V. Aspectos orofaciais dos maus-tratos infantis e da negligência odontológica. Ciênc Saúde Coletiva. 15(2):403-10.

17. Coelho FJ, Franzin LCS. Violência doméstica infanto-juvenil: importância deste conhecimento pelo profissional de saúde. Rev UNINGÁ. 2014; 20(2):104-8.

18. Nunes AJ, Sales MCV. Violência contra crianças no cenário brasileiro. Ciênc Saúde Coletiva. 2016;21(3):871-80.

\section{Correspondência para:}

Rachel Lima Ribeiro Tinoco

e-mail: rachelrtinoco@gmail.com

Rua Marechal Deodoro 217, Bloco A - Centro 24030-060 Niterói/RJ 